\title{
Remarks on Convergence among Picard, Mann and Ishikawa Iteration for Complex Space
}

\author{
Rajeshri Rana \\ Asst. Professor \\ Applied Science and Humanities \\ Department \\ G. B. Pant Engg. College, \\ Pauri Garhwal, India
}

\author{
R.C.Dimri \\ Professor \\ Department of Mathematics \\ Birla Campus \\ H.N.B. Garhwal Central Univ. \\ Srinagar-Garhwal \\ India
}

\author{
Anita Tomar \\ Asst. Professor \\ Department of Mathematics
}

Govt. Degree College Dakpathar

Vikasnagar Dehradun

India

\begin{abstract}
In this paper we have drawn a comparative analysis among convergence of Picard, Mann and Ishikawa iteration for the complex space.
\end{abstract}

\section{Keywords}

Picard iteration, Mann iteration, Ishikawa iteration

\section{INTRODUCTION}

Several authors $[1,2,4,8,9,10]$ have studied the convergence of the well known Picard's, Mann and Ishikawa iteration and have drawn different results in their comparative analysis. Many of them have declared the convergence of Mann iteration as relatively faster than Ishikawa iteration.

Our research presents an exciting result that is entirely different from the previously declared results in this field.

We have undergone a comparative analysis among Picard, Mann and Ishikawa iteration and by using MatLab programme, we found that Picard's iteration converges faster than the rest, followed by Ishikawa while Mann iteration converges slowly.

\section{PRELIMINEARIES}

Let $X$ be the metric space of complex numbers, $D$ be nonempty convex subset of $X$ and $T$ be a selfmap of $D$, let $x_{0} \in D$ [9].

The Picard's iteration is defined by

$$
x_{n+1}=T x_{n}, \quad n \geq 0
$$

The Mann iteration [6] is defined by

$$
\begin{gathered}
x_{n+1}=(1-s) x_{n}+s . T x_{n}, n \geq 0 \\
\text { where } 0<s<1
\end{gathered}
$$

The Ishikawa iteration [5] is defined by

$$
\begin{aligned}
& y_{n}=\left(1-s^{\prime}\right) x_{n}+s^{\prime} . T x_{n}, n \geq 0 \\
& x_{n+1}=(1-s) x_{n}+s . T y_{n}, n \geq 0 \\
& \quad \text { where } 0<s<1 \text { and } 0<s^{\prime}<1
\end{aligned}
$$

Obviously for $s=1$, Mann iteration reduces to Picard's iteration and for $s^{\prime}=0$ the Ishikawa iteration reduces to Mann Iteration.

In this paper, we have taken values of $s$ and $s^{\prime}$ as $0<s<1$ and $0<s^{\prime} \leq \frac{1}{2}$ for our comparative analysis and have derived fixed points for quadratic, cubic and biquadratic polynomials. Here we have the following functions:

$$
\begin{aligned}
& \text { Quadratic functions: } f(z)=z^{2}+c \\
& \text { Cubic function: } f(z)=z^{3}+c \\
& \text { Biquadractic function: } f(z)=z^{4}+c
\end{aligned}
$$

Starting with $z=(0,0)$ and $c=0.1$, we have the following reults

\section{FIXED POINTS}

3.1 Fixed points of quadratic polynomial

Table 1: Picard iteration

\begin{tabular}{|c|c|c|c|}
\hline $\begin{array}{l}\text { Number of } \\
\text { iteration } \boldsymbol{i}\end{array}$ & $|\mathbf{F}(\mathbf{z})|$ & $\begin{array}{l}\text { Number of } \\
\text { iteration } \boldsymbol{i}\end{array}$ & $|\mathbf{F}(\mathbf{z})|$ \\
\hline 1. & 0 & 6. & 0.11267 \\
\hline 2. & 0.1 & 7. & 0.11269 \\
\hline 3. & 0.11 & 8. & 0.1127 \\
\hline 4. & 0.1121 & 9. & 0.1127 \\
\hline 5. & 0.11257 & 10. & 0.1127 \\
\hline
\end{tabular}

Here we observe that the value converges to a fixed point after 07 iterations 
Figure 1: Picard iteration

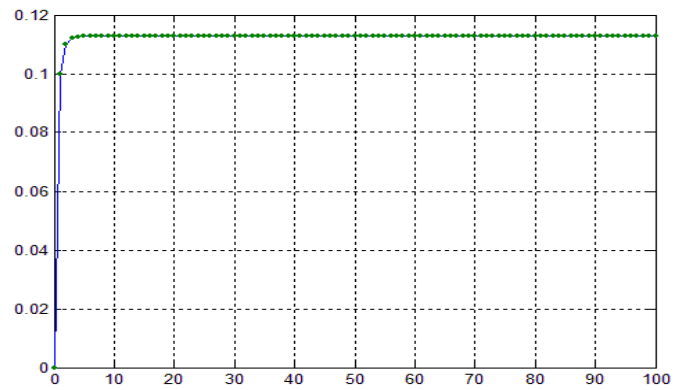

Table 2: Manniteration for $s=0.1$

\begin{tabular}{|c|c|c|c|}
\hline $\begin{array}{c}\text { Number of } \\
\text { iteration } \mathbf{i}\end{array}$ & $|\mathbf{F}(\mathbf{z})|$ & $\begin{array}{c}\text { Number of } \\
\text { iteration } \mathbf{~}\end{array}$ & $|\mathbf{F}(\mathbf{z})|$ \\
\hline 102. & 0.11267 & 112. & 0.11269 \\
\hline 103. & 0.11268 & 113. & 0.11269 \\
\hline 104. & 0.11268 & 114. & 0.11269 \\
\hline 105. & 0.11268 & 115. & 0.11269 \\
\hline 106. & 0.11268 & 116. & 0.11269 \\
\hline 107. & 0.11268 & 117. & 0.11269 \\
\hline 108. & 0.11268 & 118. & 0.11269 \\
\hline 109. & 0.11269 & 119. & 0.11269 \\
\hline 110. & 0.11269 & 120. & 0.1127 \\
\hline 111. & 0.11269 & 121. & 0.1127 \\
\hline
\end{tabular}

Here we skipped 101iteration and the value converges to a fixed point after 119 iterations

Figure 2: Mann iteration for $s=0.1$

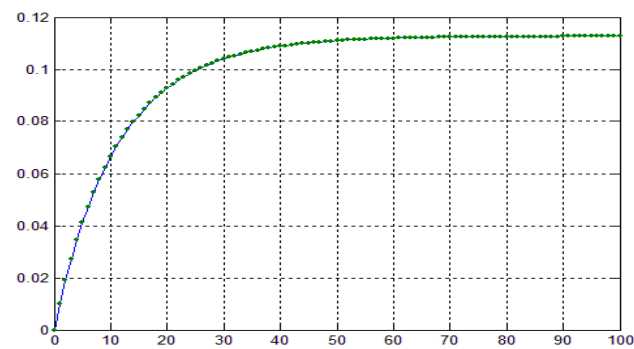

Table 3: Ishikawa iteration for $\mathrm{s}=\mathbf{0 . 6}, \mathrm{s}^{\prime}=\mathbf{0 . 1}$

\begin{tabular}{|c|c|c|c|}
\hline $\begin{array}{c}\text { Number of } \\
\text { iteration i }\end{array}$ & $|\mathbf{F}(\mathbf{z})|$ & $\begin{array}{c}\text { Number of } \\
\text { iteration i }\end{array}$ & $|\mathbf{F}(\mathbf{z})|$ \\
\hline 61. & 0.34539 & 71. & 0.34547 \\
\hline 62. & 0.3454 & 72. & 0.34547 \\
\hline 63. & 0.34542 & 73. & 0.34547 \\
\hline 64. & 0.34542 & 74. & 0.34548 \\
\hline 65. & 0.34543 & 75. & 0.34548 \\
\hline 66. & 0.34544 & 76. & 0.34548 \\
\hline 67. & 0.34545 & 77. & 0.34548 \\
\hline 68. & 0.34545 & 78. & 0.34549 \\
\hline 69. & 0.34546 & 79. & 0.34549 \\
\hline 70. & 0.34546 & 80. & 0.34549 \\
\hline
\end{tabular}

Here we skipped 60 iteration and the value converges to a fixed point after 77 iterations
Figure 3: Ishikawa iteration for $\mathrm{s}=0.6, \mathrm{~s}^{\prime}=\mathbf{0 . 1}$

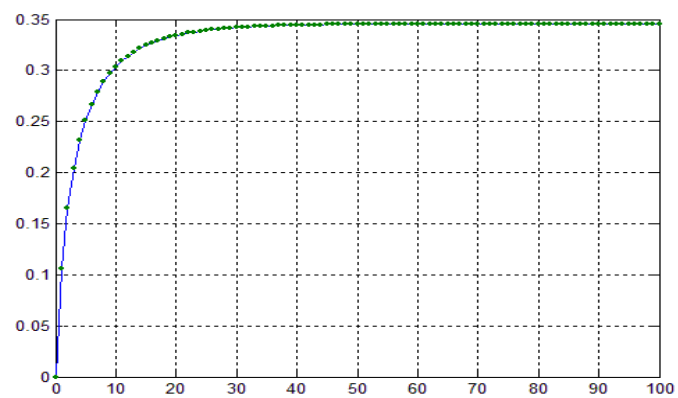

Table 4: Mann iteration fors $=0.3$

\begin{tabular}{|c|c|c|c|}
\hline $\begin{array}{l}\text { Number of } \\
\text { iteration } \mathbf{i}\end{array}$ & $|\mathbf{F}(\mathbf{z})|$ & $\begin{array}{c}\text { Number of } \\
\text { iteration } \mathbf{i}\end{array}$ & $|\mathbf{F}(\mathbf{z})|$ \\
\hline 20. & 0.11208 & 30. & 0.11266 \\
\hline 21. & 0.11223 & 31. & 0.11267 \\
\hline 22. & 0.11234 & 32. & 0.11268 \\
\hline 23. & 0.11242 & 33. & 0.11268 \\
\hline 24. & 0.11249 & 34. & 0.11269 \\
\hline 25. & 0.11254 & 35. & 0.11269 \\
\hline 26. & 0.11257 & 36. & 0.11269 \\
\hline 27. & 0.1126 & 37. & 0.11269 \\
\hline 28. & 0.11263 & 38. & 0.1127 \\
\hline 29. & 0.11264 & 39. & 0.1127 \\
\hline
\end{tabular}

Here we skipped 20 iteration and the value converges to a fixed point after 37 iterations

Figure 4: Mann iteration for $\mathrm{s}=\mathbf{0 . 3}$

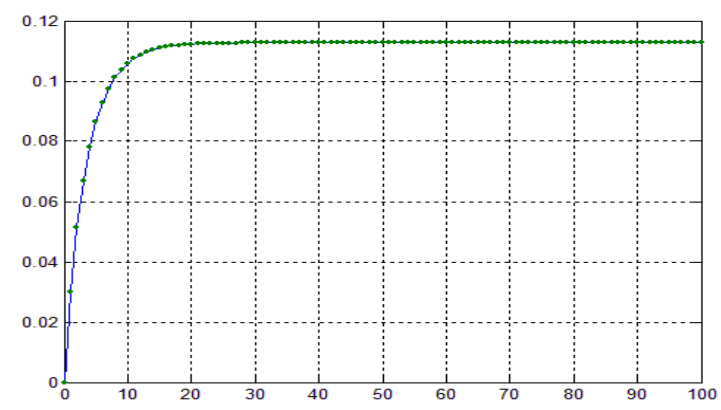

Table 5: Ishikawa iteration for $s=0.6, s^{\prime}=0.3$

\begin{tabular}{|c|r|c|c|}
\hline $\begin{array}{c}\text { Number of } \\
\text { iteration } \mathbf{i}\end{array}$ & $|\mathbf{F}(\mathbf{z})|$ & $\begin{array}{l}\text { Number of } \\
\text { iteration } \mathbf{i}\end{array}$ & $|\mathbf{F}(\mathbf{z})|$ \\
\hline 14. & 0.25432 & 24. & 0.25592 \\
\hline 15. & 0.2548 & 25. & 0.25593 \\
\hline 16. & 0.25515 & 26. & 0.25594 \\
\hline 17. & 0.25539 & 27. & 0.25595 \\
\hline 18. & 0.25556 & 28. & 0.25596 \\
\hline 19. & 0.25568 & 29. & 0.25596 \\
\hline 20. & 0.25576 & 30. & 0.25596 \\
\hline
\end{tabular}




\begin{tabular}{|r|r|l|l|}
\hline 21. & 0.25582 & 31. & 0.25596 \\
\hline 22. & 0.25587 & 32. & 0.25597 \\
\hline 23. & 0.2559 & 33. & 0.25597 \\
\hline
\end{tabular}

Here we skipped 13 iteration and the value converges to a fixed point after 31 iterations

Figure 5: Ishikawa iteration for $s=0.6, s^{\prime}=0.3$

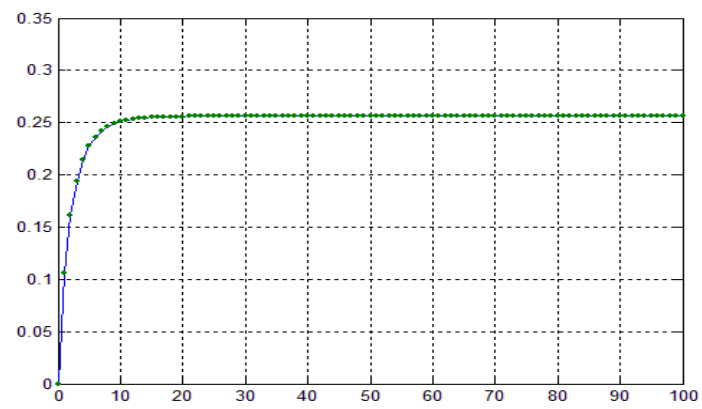

Table 6: Mann iteration for $s=0.5$

\begin{tabular}{|c|l|c|l|}
\hline $\begin{array}{l}\text { Number of } \\
\text { iteration i }\end{array}$ & $|\mathrm{F}(\mathrm{z})|$ & $\begin{array}{l}\text { Number of } \\
\text { iteration i }\end{array}$ & $|\mathrm{F}(\mathrm{z})|$ \\
\hline 4. & 0.091032 & 14. & 0.11255 \\
\hline 5. & 0.099659 & 15. & 0.11261 \\
\hline 6. & 0.1048 & 16. & 0.11264 \\
\hline 7. & 0.10789 & 17. & 0.11267 \\
\hline 8. & 0.10976 & 18. & 0.11268 \\
\hline 9. & 0.11091 & 19. & 0.11269 \\
\hline 10. & 0.1116 & 20. & 0.11269 \\
\hline 11. & 0.11203 & 21. & 0.1127 \\
\hline 12. & 0.11229 & 22. & 0.1127 \\
\hline 13. & 0.11245 & 23. & 0.1127 \\
\hline
\end{tabular}

Here we skipped 03 iteration and the value converges to a fixed point after 20 iterations

Figure 6: Mann iteration for $s=0.5$

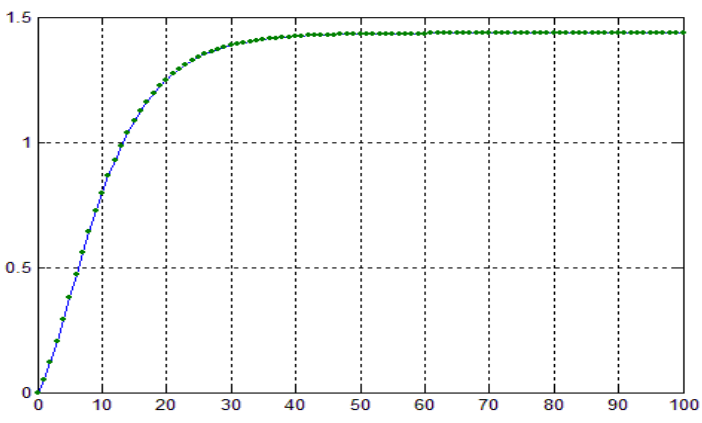

Table 7: Ishikawa iteration for $s^{=0.6}, s^{\prime}=0.5$

\begin{tabular}{|c|l|c|l|}
\hline $\begin{array}{c}\text { Number of } \\
\text { iteration i }\end{array}$ & $|\mathbf{F}(\mathbf{z})|$ & $\begin{array}{l}\text { Number of } \\
\text { iteration } \mathbf{i}\end{array}$ & $|\mathbf{F}(\mathbf{z})|$ \\
\hline 4. & 0.18492 & 14. & 0.22192 \\
\hline 5. & 0.20032 & 15. & 0.22207 \\
\hline 6. & 0.20923 & 16. & 0.22216 \\
\hline 7. & 0.21447 & 17. & 0.22221 \\
\hline 8. & 0.21759 & 18. & 0.22224 \\
\hline 9. & 0.21946 & 19. & 0.22226 \\
\hline 10. & 0.22059 & 20. & 0.22228 \\
\hline 11. & 0.22126 & 21. & 0.22228 \\
\hline 12. & 0.22167 & 22. & 0.22229 \\
\hline 13. & 0.18492 & 23. & 0.22229 \\
\hline
\end{tabular}

Here we skipped 03 iteration and the value converges to a fixed point after 21 iterations

Figure 7: Ishikawa iteration for $s=0.6, s^{\prime}=0.5$

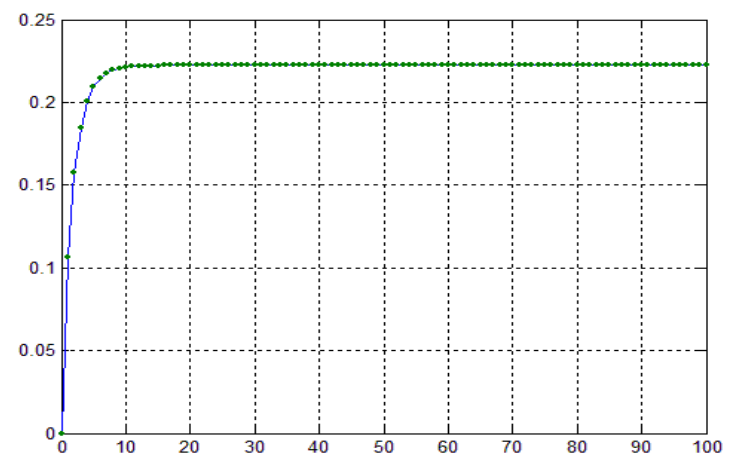

Table 8: Mann iteration for $s=0.8$

\begin{tabular}{|c|c|c|c|}
\hline $\begin{array}{l}\text { Number of } \\
\text { iteration i }\end{array}$ & $|\mathbf{F}(\mathbf{z})|$ & $\begin{array}{l}\text { Number of } \\
\text { iteration i }\end{array}$ & $|\mathbf{F}(\mathbf{z})|$ \\
\hline 1. & 0 & 11. & 0.1127 \\
\hline 2. & 0.08 & 12. & 0.1127 \\
\hline 3. & 0.10112 & 13. & 0.1127 \\
\hline 4. & 0.1084 & 14. & 0.1127 \\
\hline 5. & 0.11108 & 15. & 0.1127 \\
\hline 6. & 0.11209 & 16. & 0.1127 \\
\hline 7. & 0.11247 & 17. & 0.1127 \\
\hline 8. & 0.11261 & 18. & 0.1127 \\
\hline 9. & 0.11267 & 19. & 0.1127 \\
\hline 10. & 0.11269 & 20. & 0.1127 \\
\hline
\end{tabular}

Here we observe that the value converges to a fixed point after 11 iterations 
Figure 8: Mann iteration for $s=0.8$

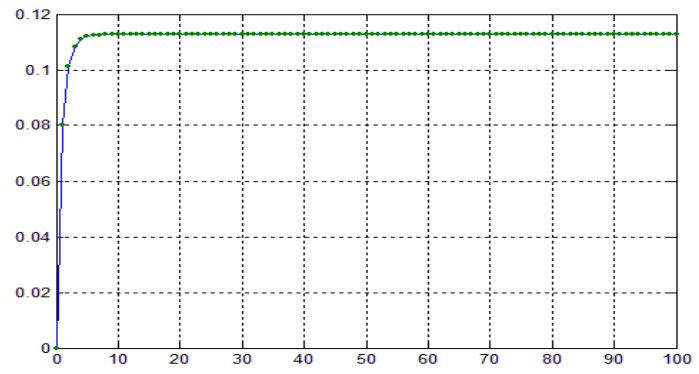

Table 9: Ishikawa iteration for $s=0.6, s^{\prime}=0.8$

\begin{tabular}{|c|l|c|l|}
\hline $\begin{array}{l}\text { Number of } \\
\text { iteration i }\end{array}$ & $|\mathbf{F}(\mathbf{z})|$ & $\begin{array}{l}\text { Number of } \\
\text { iteration } \mathbf{i}\end{array}$ & $|\mathbf{F}(\mathbf{z})|$ \\
\hline 5. & 0.18496 & 15. & 0.19544 \\
\hline 6. & 0.19019 & 16. & 0.19544 \\
\hline 7. & 0.1928 & 17. & 0.19544 \\
\hline 8. & 0.19412 & 18. & 0.19545 \\
\hline 9. & 0.19478 & 19. & $1.95 \mathrm{E}-01$ \\
\hline 10. & 0.19511 & 20. & $1.95 \mathrm{E}-01$ \\
\hline 11. & 0.19528 & 21. & $1.95 \mathrm{E}-01$ \\
\hline 12. & 0.19536 & 22. & 0.19545 \\
\hline 13. & 0.1954 & 23. & 0.19545 \\
\hline 14. & 0.19543 & 24. & 0.19545 \\
\hline
\end{tabular}

Here we skipped 04 iteration and the value converges to a fixed point after 21 iterations

Figure 9: Ishikawa iteration for $s=0.6, s^{\prime}=0.8$

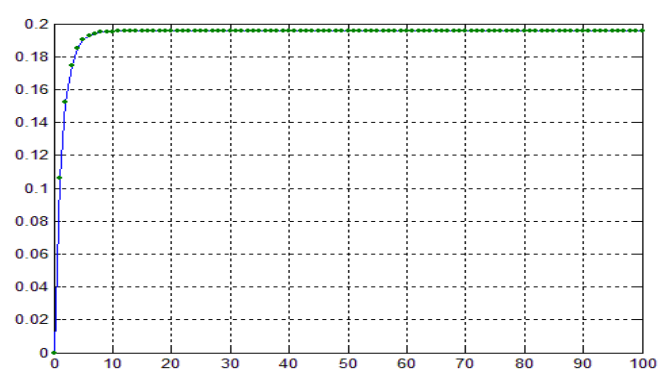

\subsection{Fixed points of Cubic polynomial}

Table 10: Picard iteration

\begin{tabular}{|c|c|c|c|}
\hline $\begin{array}{l}\text { Number of } \\
\text { iteration } i\end{array}$ & $|\mathbf{F}(\mathbf{z})|$ & $\begin{array}{l}\text { Number of } \\
\text { iteration } i\end{array}$ & $|\mathbf{F}(\mathbf{z})|$ \\
\hline 1. & 0 & 6. & 0.10103 \\
\hline 2. & 0.1 & 7. & 0.10103 \\
\hline 3. & 0.101 & 8. & 0.10103 \\
\hline 4. & 0.10103 & 9. & 0.10103 \\
\hline 5. & 0.10103 & 10. & 0.10103 \\
\hline
\end{tabular}

Here we observe that the value converges to a fixed point after 03 iterations
Figure 10: Picard iteration

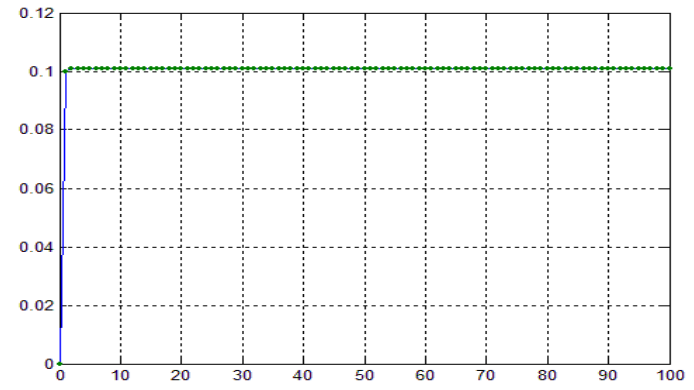

Table 11: Mann iteration for $s=0.1$

\begin{tabular}{|c|c|c|c|}
\hline $\begin{array}{l}\text { Number of } \\
\text { iteration i }\end{array}$ & $|\mathbf{F}(\mathbf{z})|$ & $\begin{array}{l}\text { Number of } \\
\text { iteration i }\end{array}$ & $|\mathbf{F}(\mathbf{z})|$ \\
\hline 79. & 0.101 & 89. & 0.10102 \\
\hline 80. & 0.101 & 90. & 0.10102 \\
\hline 81. & 0.101 & 91. & 0.10102 \\
\hline 82. & 0.10101 & 92. & 0.10102 \\
\hline 83. & 0.10101 & 93. & 0.10102 \\
\hline 84. & 0.10101 & 94. & 0.10102 \\
\hline 85. & 0.10101 & 95. & 0.10102 \\
\hline 86. & 0.10101 & 96. & 0.10103 \\
\hline 87. & 0.10102 & 97. & 0.10103 \\
\hline 88. & 0.10102 & 98. & 0.10103 \\
\hline
\end{tabular}

Here we skipped 78 iteration and the value converges to a fixed point after 95 iterations

Figure 11: Mann iteration for $\mathrm{s}=\mathbf{0 . 1}$

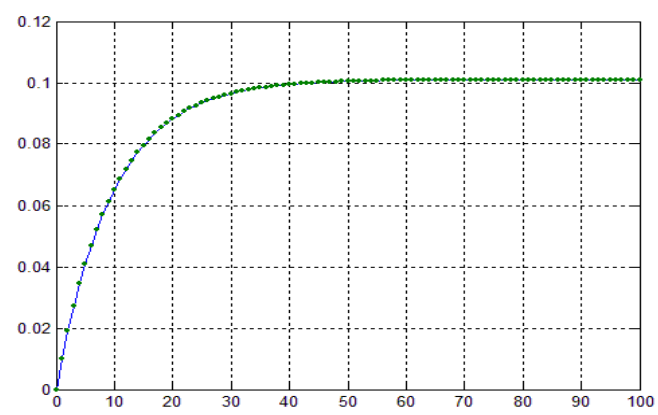

Table 12: Ishikawa iteration for $s=0.6, s^{\prime}=0.1$

\begin{tabular}{|c|c|c|c|}
\hline $\begin{array}{l}\text { Number of } \\
\text { iteration } \mathbf{i}\end{array}$ & $|\mathbf{F}(\mathbf{z})|$ & $\begin{array}{l}\text { Number of } \\
\text { iteration } \mathbf{i}\end{array}$ & $|\mathbf{F}(\mathbf{z})|$ \\
\hline 1. & 0 & 11. & 0.18571 \\
\hline 2. & 0.1006 & 12. & 0.1858 \\
\hline 3. & 0.1444 & 13. & 0.18585 \\
\hline 4. & 0.16508 & 14. & 0.18588 \\
\hline 5. & 0.1753 & 15. & 0.18589 \\
\hline
\end{tabular}




\begin{tabular}{|c|c|c|c|}
\hline 6. & 0.18046 & 16. & 0.1859 \\
\hline 7. & 0.1831 & 17. & 0.1859 \\
\hline 8. & 0.18446 & 18. & 0.1859 \\
\hline 9. & 0.18516 & 19. & 0.18591 \\
\hline 10. & 0.18552 & 20. & 0.18591 \\
\hline
\end{tabular}

Here we observe that the value converges to a fixed point after 19 iterations

Figure 12: Ishikawa iteration for $s=0.6, s^{\prime}=0.1$

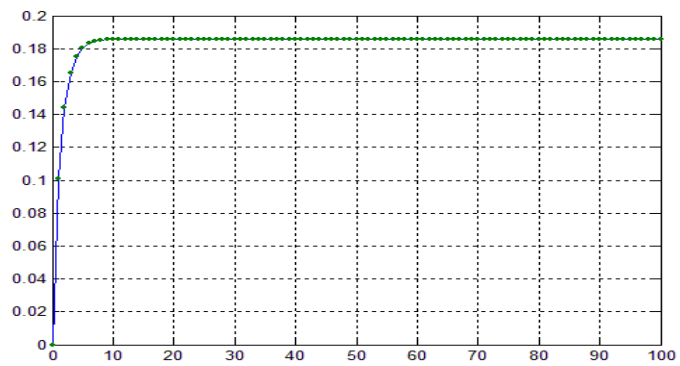

Table 13: Mann iteration for $s=0.3$

\begin{tabular}{|c|c|c|c|}
\hline $\begin{array}{c}\text { Number of } \\
\text { iteration } \mathbf{i}\end{array}$ & $|\mathbf{F}(\mathbf{z})|$ & $\begin{array}{c}\text { Number of } \\
\text { iteration } \mathbf{i}\end{array}$ & $|\mathbf{F}(\mathbf{z})|$ \\
\hline 12. & 0.098804 & 22. & 0.10096 \\
\hline 13. & 0.099452 & 23. & 0.10098 \\
\hline 14. & 0.099911 & 24. & 0.101 \\
\hline 15. & 0.10024 & 25. & 0.10101 \\
\hline 16. & 0.10047 & 26. & 0.10101 \\
\hline 17. & 0.10063 & 27. & 0.10102 \\
\hline 18. & 0.10075 & 28. & 0.10102 \\
\hline 19. & 0.10083 & 29. & 0.10102 \\
\hline 20. & 0.10089 & 30. & 0.10103 \\
\hline 21. & 0.10093 & 31. & 0.10103 \\
\hline
\end{tabular}

Here we skipped 11 iterations and the value converges to a fixed point after 29 iterations

Figure 13: Mann iteration for $s=0.3$

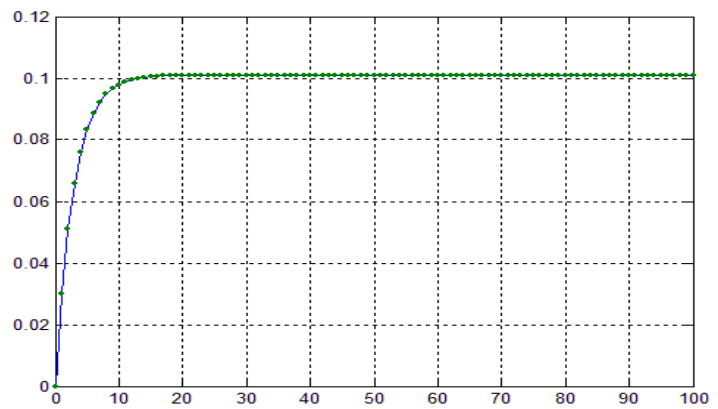

Table 14: Ishikawa iteration for $s=0.6, s^{\prime}=0.3$

\begin{tabular}{|c|c|c|c|}
\hline $\begin{array}{c}\text { Number of } \\
\text { iteration } \mathbf{i}\end{array}$ & $|\mathbf{F}(\mathbf{z})|$ & $\begin{array}{c}\text { Number of } \\
\text { iteration } \mathbf{i}\end{array}$ & $|\mathbf{F}(\mathbf{z})|$ \\
\hline 1. & 0 & 11. & 0.17821 \\
\hline 2. & 0.1006 & 12. & 0.17825 \\
\hline 3. & 0.14323 & 13. & 0.17827 \\
\hline 4. & 0.16217 & 14. & 0.17828 \\
\hline 5. & 0.17082 & 15. & 0.17828 \\
\hline 6. & 0.17481 & 16. & 0.17829 \\
\hline 7. & 0.17666 & 17. & 0.17829 \\
\hline 8. & 0.17753 & 18. & 0.17829 \\
\hline 9. & 0.17793 & 19. & 0.17829 \\
\hline 10. & 0.17812 & 20. & 0.17829 \\
\hline
\end{tabular}

Here we observe that the value converges to a fixed point after 15 iterations

Figure 14: Ishikawa iteration for $s=0.6, s^{\prime}=0.3$

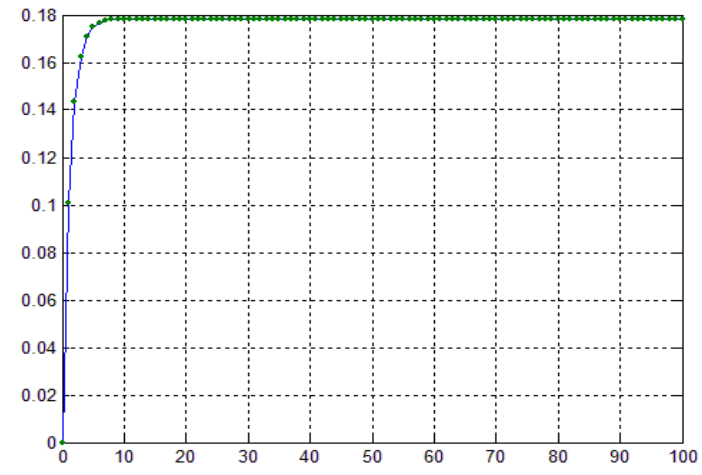

Table 15: Mann iteration for $s=0.5$

\begin{tabular}{|c|c|c|c|}
\hline $\begin{array}{l}\text { Number of } \\
\text { iteration i }\end{array}$ & $|\mathbf{F}(\mathbf{z})|$ & $\begin{array}{c}\text { Number of } \\
\text { iteration i }\end{array}$ & $|\mathbf{F}(\mathbf{z})|$ \\
\hline 1. & 0 & 11. & 0.1009 \\
\hline 2. & 0.05 & 12. & 0.10097 \\
\hline 3. & 0.075063 & 13. & 0.101 \\
\hline 4. & 0.087743 & 14. & 0.10101 \\
\hline 5. & 0.094209 & 15. & 0.10102 \\
\hline 6. & 0.097523 & 16. & 0.10103 \\
\hline 7. & 0.099225 & 17. & 0.10103 \\
\hline 8. & 0.1001 & 18. & 0.10103 \\
\hline 9. & 0.10055 & 19. & 0.10103 \\
\hline 10. & 0.10078 & 20. & 0.10103 \\
\hline
\end{tabular}

Here we observe that the value converges to a fixed point after 15 iterations 
Figure 15: Mann iteration for $s=0.5$

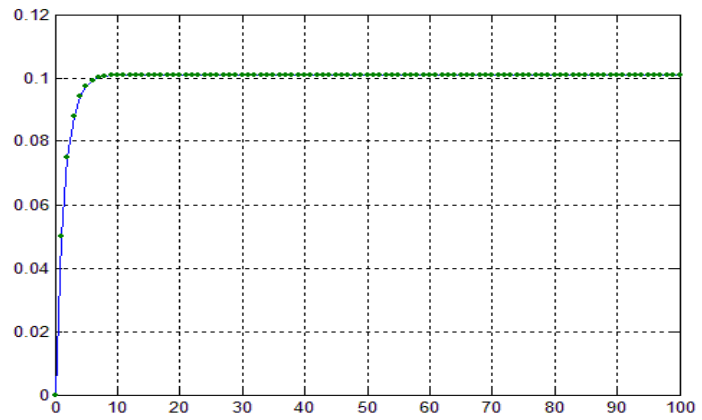

Table 16: Ishikawa iteration for $s=0.6, s^{\prime}=0.5$

\begin{tabular}{|c|c|c|c|}
\hline $\begin{array}{l}\text { Number of } \\
\text { iteration i }\end{array}$ & $|\mathbf{F}(\mathbf{z})|$ & $\begin{array}{l}\text { Number of } \\
\text { iteration } \mathbf{i}\end{array}$ & $|\mathbf{F}(\mathbf{z})|$ \\
\hline 1. & 0 & 11. & 0.17341 \\
\hline 2. & 0.1006 & 12. & 0.17344 \\
\hline 3. & 0.1423 & 13. & 0.17345 \\
\hline 4. & 0.16 & 14. & 0.17345 \\
\hline 5. & 0.16762 & 15. & 0.17345 \\
\hline 6. & 0.17092 & 16. & 0.17345 \\
\hline 7. & 0.17235 & 17. & 0.17345 \\
\hline 8. & 0.17297 & 18. & 0.17345 \\
\hline 9. & 0.17325 & 19. & 0.17345 \\
\hline 10. & 0.17336 & 20. & 0.17345 \\
\hline
\end{tabular}

Here we observe that the value converges to a fixed point after 13 iterations

Figure 16: Ishikawa iteration for $s=0.6, s^{\prime}=0.5$

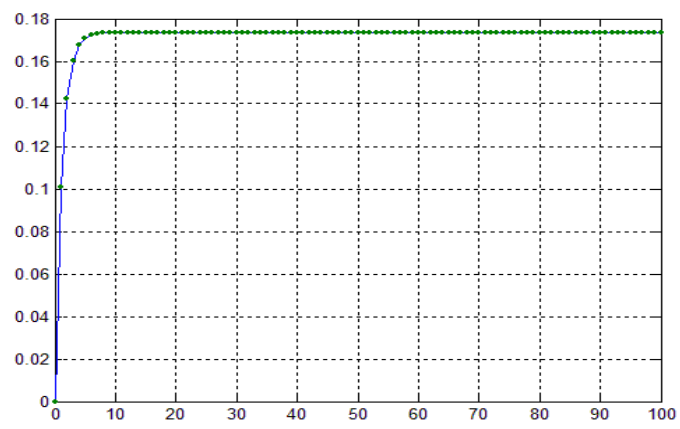

Table 17: Mann iteration for $s=0.8$

\begin{tabular}{|c|c|c|c|}
\hline $\begin{array}{l}\text { Number of } \\
\text { iteration i }\end{array}$ & $|\mathbf{F}(\mathbf{z})|$ & $\begin{array}{l}\text { Number of } \\
\text { iteration } \mathbf{i}\end{array}$ & $|\mathbf{F}(\mathbf{z})|$ \\
\hline 1. & 0 & 11. & 0.10103 \\
\hline 2. & 0.08 & 12. & 0.10103 \\
\hline 3. & 0.09641 & 13. & 0.10103 \\
\hline 4. & 0.099999 & 14. & 0.10103 \\
\hline
\end{tabular}

\begin{tabular}{|c|c|c|c|}
\hline 5. & 0.1008 & 15. & 0.10103 \\
\hline 6. & 0.10098 & 16. & 0.10103 \\
\hline 7. & 0.10102 & 17. & 0.10103 \\
\hline 8. & 0.10103 & 18. & 0.10103 \\
\hline 9. & 0.10103 & 19. & 0.10103 \\
\hline 10. & 0.10103 & 20. & 0.10103 \\
\hline
\end{tabular}

Here we observe that the value converges to a fixed point after 07 iterations

Figure 17: Mann iteration for $\mathrm{s}=\mathbf{0 . 8}$

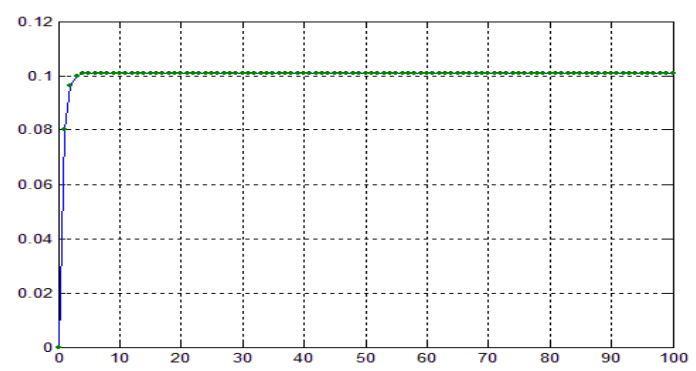

Table 18: Ishikawa iteration for $s=0.6, s^{\prime}=0.8$

\begin{tabular}{|c|c|c|l|}
\hline $\begin{array}{c}\text { Number of } \\
\text { iteration i }\end{array}$ & $|\mathbf{F}(\mathbf{z})|$ & $\begin{array}{c}\text { Number of } \\
\text { iteration } \mathbf{i}\end{array}$ & $|\mathbf{F}(\mathbf{z})|$ \\
\hline 1. & 0 & 11. & 0.16926 \\
\hline 2. & 0.1006 & 12. & 0.16927 \\
\hline 3. & 0.1413 & 13. & 0.16928 \\
\hline 4. & 0.15785 & 14. & 0.16928 \\
\hline 5. & 0.16461 & 15. & 0.16928 \\
\hline 6. & 0.16737 & 16. & 0.16928 \\
\hline 7. & 0.1685 & 17. & 0.16928 \\
\hline 8. & 0.16896 & 18. & 0.16928 \\
\hline 9. & 0.16915 & 19. & 0.16928 \\
\hline 10. & 0.16923 & 20. & 0.16928 \\
\hline
\end{tabular}

Here we observe that the value converges to a fixed point after 12 iterations

Figure 18: Ishikawa iteration for $s=0.6, s^{\prime}=0.8$

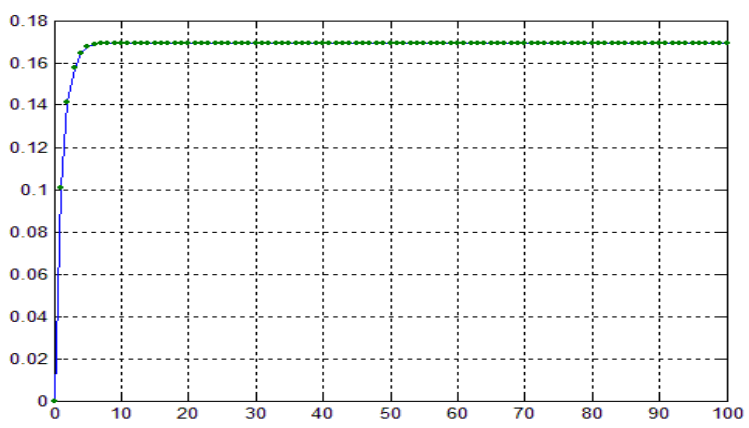




\subsection{Fixed points of Bi-quadratic polynomial}

Table 19: Picard iteration

\begin{tabular}{|c|c|c|c|}
\hline $\begin{array}{l}\text { Number of } \\
\text { iteration } \boldsymbol{i}\end{array}$ & $|\mathbf{F}(\mathbf{z})|$ & $\begin{array}{l}\text { Number of } \\
\text { iteration } \boldsymbol{i}\end{array}$ & $|\mathbf{F}(\mathbf{z})|$ \\
\hline 1. & 0 & 6. & 0.1001 \\
\hline 2. & 0.1 & 7. & 0.1001 \\
\hline 3. & 0.1001 & 8. & 0.1001 \\
\hline 4. & 0.1001 & 9. & 0.1001 \\
\hline 5. & 0.1001 & 10. & 0.1001 \\
\hline
\end{tabular}

Here we observe that the value converges to a fixed point after 02 iterations

Figure 19: Picard iteration

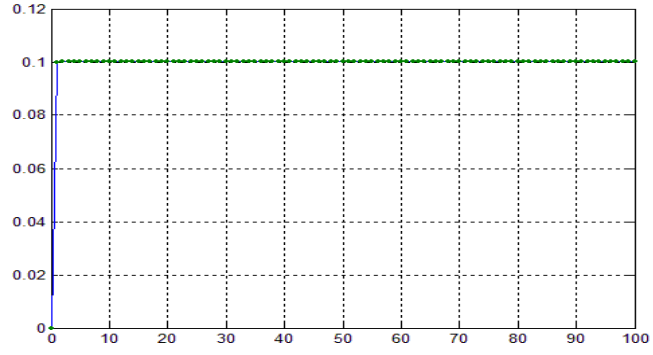

Table 20: Mann iteration for $s=0.1$

\begin{tabular}{|c|c|c|c|}
\hline $\begin{array}{l}\text { Number of } \\
\text { iteration i }\end{array}$ & $|\mathbf{F}(\mathbf{z})|$ & $\begin{array}{c}\text { Number of } \\
\text { iteration i }\end{array}$ & $|\mathbf{F}(\mathbf{z})|$ \\
\hline 77. & 0.10007 & 87. & 0.10009 \\
\hline 78. & 0.10007 & 88. & 0.10009 \\
\hline 79. & 0.10007 & 89. & 0.10009 \\
\hline 80. & 0.10008 & 90. & 0.10009 \\
\hline 81. & 0.10008 & 91. & 0.10009 \\
\hline 82. & 0.10008 & 92. & 0.10009 \\
\hline 83. & 0.10008 & 93. & 0.10009 \\
\hline 84. & 0.10008 & 94. & 0.10009 \\
\hline 85. & 0.10009 & 95. & 0.1001 \\
\hline 86. & 0.10009 & 96. & 0.1001 \\
\hline
\end{tabular}

Here we skipped 76 iteration and the value converges to a fixed point after 95 iterations

Figure 20: Mann iteration for $\mathrm{s}=\mathbf{0 . 1}$

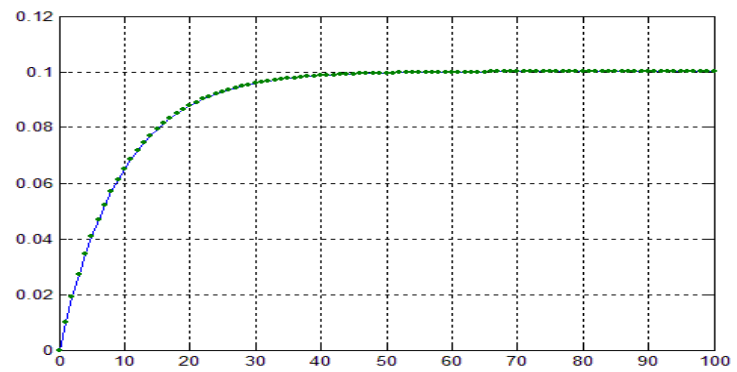

Table 21: Ishikawa iteration for $\mathrm{s}=\mathbf{0 . 6}, \mathrm{s}^{\prime}=\mathbf{0 . 1}$

\begin{tabular}{|c|c|c|c|}
\hline $\begin{array}{l}\text { Number of } \\
\text { iteration i }\end{array}$ & $|\mathbf{F}(\mathbf{z})|$ & $\begin{array}{l}\text { Number of } \\
\text { iteration i }\end{array}$ & $|\mathbf{F}(\mathbf{z})|$ \\
\hline 1. & 0 & 11. & 0.17078 \\
\hline 2. & 0.10006 & 12. & 0.1708 \\
\hline 3. & 0.14081 & 13. & 0.17081 \\
\hline 4. & 0.15791 & 14. & 0.17081 \\
\hline 5. & 0.16523 & 15. & 0.17082 \\
\hline 6. & 0.16839 & 16. & 0.17082 \\
\hline 7. & 0.16976 & 17. & 0.17082 \\
\hline 8. & 0.17036 & 18. & 0.17082 \\
\hline 9. & 0.17062 & 19. & 0.17082 \\
\hline 10. & 0.17073 & 20. & 0.17082 \\
\hline
\end{tabular}

Here we observe that the value converges to a fixed point after 14 iterations

Figure 21: Ishikawa iteration for $s^{=0.6}, s^{\prime}=0.1$

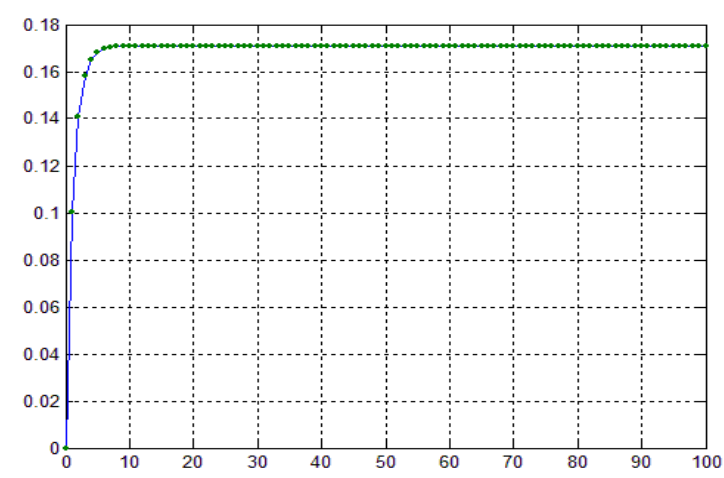

Table 22: Mann iteration for $s=0.3$

\begin{tabular}{|c|c|c|c|}
\hline $\begin{array}{l}\text { Number of } \\
\text { iteration i }\end{array}$ & $|\mathbf{F}(\mathbf{z})|$ & $\begin{array}{l}\text { Number of } \\
\text { iteration i }\end{array}$ & $|\mathbf{F}(\mathbf{z})|$ \\
\hline 11. & 0.09724 & 21. & 0.10002 \\
\hline 12. & 0.098095 & 22. & 0.10004 \\
\hline 13. & 0.098694 & 23. & 0.10006 \\
\hline 14. & 0.099114 & 24. & 0.10007 \\
\hline 15. & 0.099409 & 25. & 0.10008 \\
\hline 16. & 0.099616 & 26. & 0.10009 \\
\hline 17. & 0.09976 & 27. & 0.10009 \\
\hline 18. & 0.099862 & 28. & 0.10009 \\
\hline 19. & 0.099933 & 29. & 0.1001 \\
\hline 20. & 0.099983 & 30. & 0.1001 \\
\hline
\end{tabular}

Here we skipped 10 iterations and the value converges to a fixed point after 28 iterations 
Figure 22: Mann iteration for $\mathrm{s}=\mathbf{0 . 3}$

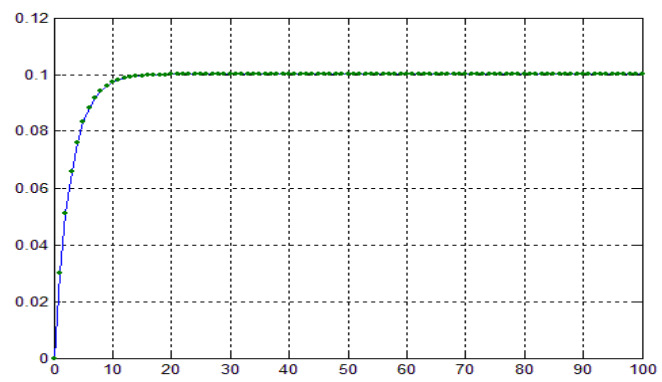

Table 23: Ishikawa iteration for $s=0.6, s^{\prime}=0.3$

\begin{tabular}{|c|c|c|c|}
\hline $\begin{array}{l}\text { Number } \\
\text { of } \\
\text { iteration i }\end{array}$ & $|\mathbf{F}(\mathbf{z})|$ & $\begin{array}{l}\text { Number } \\
\text { of } \\
\text { iteration i }\end{array}$ & $|\mathbf{F}(\mathbf{z})|$ \\
\hline 1. & 0 & 11. & 0.16892 \\
\hline 2. & 0.10006 & 12. & 0.16894 \\
\hline 3. & 0.14053 & 13. & 0.16894 \\
\hline 4. & 0.15714 & 14. & 0.16894 \\
\hline 5. & 0.16403 & 15. & 0.16895 \\
\hline 6. & 0.16689 & 16. & 0.16895 \\
\hline 7. & 0.16809 & 17. & 0.16895 \\
\hline 8. & 0.16859 & 18. & 0.16895 \\
\hline 9. & 0.1688 & 19. & 0.16895 \\
\hline 10. & 0.16888 & 20. & 0.16895 \\
\hline
\end{tabular}

Here we observe that the value converges to a fixed point after 14 iterations

Figure 23: Ishikawa iteration for $s=0.6, s^{\prime}=0.3$

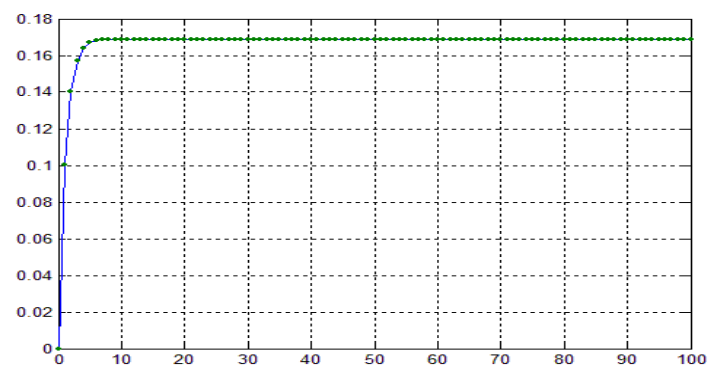

Table 24: Mann iteration for $s=0.5$

\begin{tabular}{|c|c|c|c|}
\hline $\begin{array}{l}\text { Number of } \\
\text { iteration i }\end{array}$ & $|\mathbf{F}(\mathbf{z})|$ & $\begin{array}{l}\text { Number of } \\
\text { iteration i }\end{array}$ & $|\mathbf{F}(\mathbf{z})|$ \\
\hline 1. & 0 & 11. & 0.099999 \\
\hline 2. & 0.05 & 12. & 0.10005 \\
\hline 3. & 0.075003 & 13. & $\mathbf{0 . 1 0 0 0 7}$ \\
\hline 4. & 0.087517 & 14. & 0.10009 \\
\hline 5. & 0.093788 & 15. & 0.10009 \\
\hline 6. & 0.096933 & 16. & 0.1001 \\
\hline 7. & 0.09851 & 17. & 0.1001 \\
\hline 8. & 0.099302 & 18. & 0.1001 \\
\hline
\end{tabular}

\begin{tabular}{|l|c|c|c|}
\hline 9. & 0.0997 & 19. & 0.1001 \\
\hline 10. & 0.099899 & 20. & 0.1001 \\
\hline
\end{tabular}

Here we observe that the value converges to a fixed point after 15 iterations

Figure 24: Mann iteration for $s=0.5$

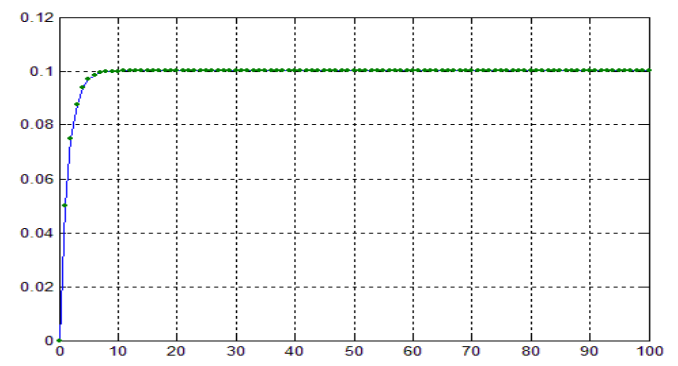

Table 25: Ishikawa iteration for $\mathrm{s}=\mathbf{0 . 6}, \mathrm{s}^{\prime}=\mathbf{0 . 5}$

\begin{tabular}{|c|c|c|c|}
\hline $\begin{array}{l}\text { Number } \\
\text { of } \\
\text { iteration i }\end{array}$ & $|\mathbf{F}(\mathbf{z})|$ & $\begin{array}{l}\text { Number } \\
\text { of } \\
\text { iteration } \mathbf{i}\end{array}$ & $|\mathbf{F}(\mathbf{z})|$ \\
\hline 1. & 0 & 11. & 0.1678 \\
\hline 2. & 0.10006 & 12. & 0.16781 \\
\hline 3. & 0.14033 & 13. & 0.16782 \\
\hline 4. & 0.15664 & 14. & 0.16782 \\
\hline 5. & 0.16327 & 15. & 0.16782 \\
\hline 6. & 0.16596 & 16. & 0.16782 \\
\hline 7. & 0.16706 & 17. & 0.16782 \\
\hline 8. & 0.16751 & 18. & 0.16782 \\
\hline 9. & 0.16769 & 19. & 0.16782 \\
\hline 10. & 0.16777 & 20. & 0.16782 \\
\hline
\end{tabular}

Here we observe that the value converges to a fixed point after 25 iterations

Figure 25: Ishikawa iteration for $s=0.6, s^{\prime}=0.5$

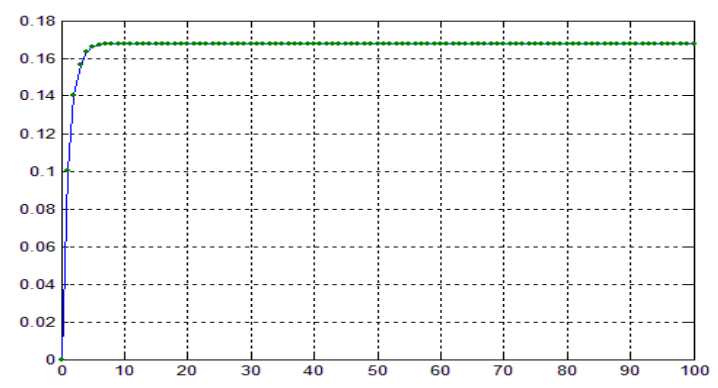

Table 26: Mann iteration for $s=0.8$

\begin{tabular}{|c|c|c|c|}
\hline $\begin{array}{l}\text { Number } \\
\text { of } \\
\text { iteration i }\end{array}$ & $|\mathbf{F}(\mathbf{z})|$ & $\begin{array}{l}\text { Number } \\
\text { of } \\
\text { iteration } \mathbf{i}\end{array}$ & $|\mathbf{F}(\mathbf{z})|$ \\
\hline 1. & 0 & 11. & 0.1001 \\
\hline 2. & 0.08 & 12. & 0.1001 \\
\hline 3. & 0.096033 & 13. & 0.1001 \\
\hline 4. & 0.099275 & 14. & 0.1001 \\
\hline 5. & 0.099933 & 15. & 0.1001 \\
\hline
\end{tabular}




\begin{tabular}{|c|c|c|c|}
\hline 6. & 0.10007 & 16. & 0.1001 \\
\hline 7. & 0.10009 & 17. & 0.1001 \\
\hline 8. & 0.1001 & 18. & 0.1001 \\
\hline 9. & 0.1001 & 19. & 0.1001 \\
\hline 10. & 0.1001 & 20. & 0.1001 \\
\hline
\end{tabular}

Here we observe that the value converges to a fixed point after 07 iterations

Figure 26: Mann iteration for $s=0.8$

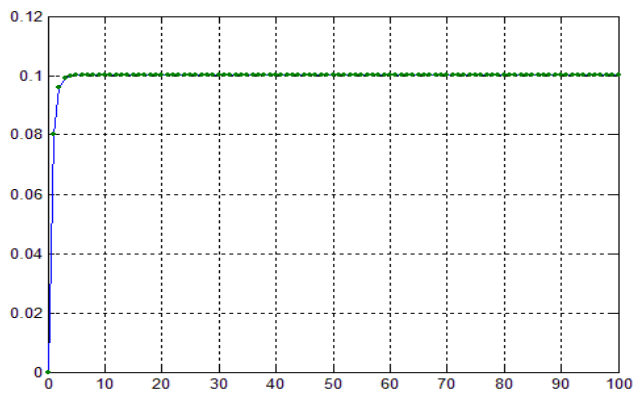

Table 27: Ishikawa iteration for $s=0.6, s^{\prime}=0.8$

\begin{tabular}{|c|c|c|c|}
\hline $\begin{array}{l}\text { Number of } \\
\text { iteration i }\end{array}$ & $|\mathbf{F}(\mathbf{z})|$ & $\begin{array}{l}\text { Number of } \\
\text { iteration i }\end{array}$ & $|\mathbf{F}(\mathbf{z})|$ \\
\hline 1. & 0 & 11. & 0.16697 \\
\hline 2. & 0.10006 & 12. & 0.16698 \\
\hline 3. & 0.14015 & 13. & 0.16699 \\
\hline 4. & 0.15622 & 14. & 0.16699 \\
\hline 5. & 0.16267 & 15. & 0.16699 \\
\hline 6. & 0.16526 & 16. & 0.16699 \\
\hline 7. & 0.16629 & 17. & 0.16699 \\
\hline 8. & 0.16671 & 18. & 0.16699 \\
\hline 9. & 0.16688 & 19. & 0.16699 \\
\hline 10. & 0.16694 & 20. & 0.16699 \\
\hline
\end{tabular}

Here we observe that the value converges to a fixed point after 12 iterations

Figure 27: Ishikawa iteration for $s=0.6, s^{\prime}=0.8$

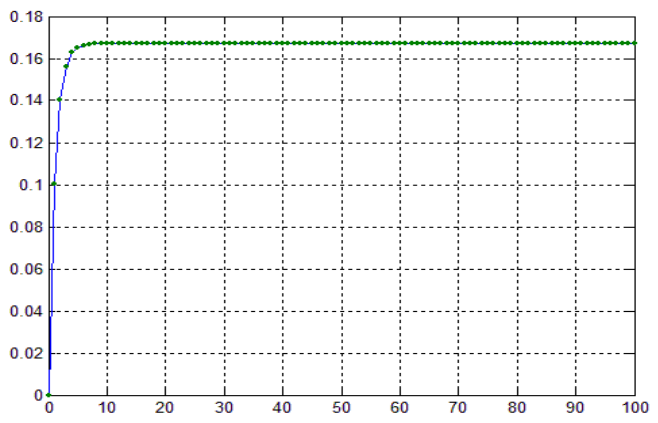

\section{COMPARATIVE ANALYSIS}

For Quadratic polynomial we observe that Picard iteration converges very fast to fixed point after 07 iterations. On the other hand for $s=0.6, s^{\prime}=0.1$, Ishikawa iteration converges faster to fixed point (after 77 iterations) as comparative to Mann iteration which converges after 119 iterations.

Similarly for $s=0.6, s^{\prime}=0.3$ Ishikawa converges after 31 iterations to fixed point while Mann iteration for $s=0.3$ converges after 37 iterations.

But for $\mathrm{s}=0.6, \mathrm{~s}^{\prime}=0.5$ Ishikawa iteration and Mann iteration for $\mathrm{s}=0.5$ shows equivalence as both converges after 20 iterations.

For Cubic polynomial we observe that Picard again converges (after 03 iterations) faster than Mann and Ishikawa iterations. On the other hand $s=0.6, s^{\prime}=0.1$, Ishikawa iteration converges to fixed point after 19 iterations which is comparatively faster than Mann iteration for $s=0.1$, which converges to fixed point after 95 iterations.

Similarly for $s=0.6, s^{\prime}=0.3$ Ishikawa iteration converges after 15 iterations while Mann iteration for $\mathrm{s}=0.3$, converges after 29 iterations.

Likewise for $\mathrm{s}=0.6, \mathrm{~s}^{\prime}=0.5$ Ishikawa iteration converges after 12 iterations while Mann iteration for $\mathrm{s}=0.5$, converges after 15 iterations.

For Biquadratic polynomial, we again observe that Picard iteration converges to fixed point very fast after 02 iterations. On the other hand, for $s=0.6, s^{\prime}=0.1$, Ishikawa iteration converges after 14 iterations while Mann iteration for $\mathrm{s}=0.1$ converges after 95 iterations.

Similarly for $s=0.6, s^{\prime}=0.3$ Ishikawa iteration converges after 14 iterations while Mann iteration for $\mathrm{s}=0.3$, converges after 28 iterations.

Likewise for $s=0.6, s^{\prime}=0.5$ Ishikawa iteration converges after 12 iterations while Mann iteration for $\mathrm{s}=0.5$, converges after 15 iterations.

In this paper we have not considered higher values of $\mathrm{s}$ because if we consider $\mathrm{s}=0.8$ or more higher, than Mann iteration starts behaving like Picard and so it will converges comparatively faster than Ishikawa iteration. Table 8 and Table 9 of 3.1, 3.2 and 3.3 shows the following behavior.

\section{CONCLUSION}

The unprecedented graphical study done previously by us shows that for the above mentioned conditions $0<s<1,0<s^{\prime} \leq \frac{1}{2}$, we have Ishikawa iteration converging faster than Mann iteration while Picard's iteration converges faster than both.

Besides this, we also observe that as the power of the polynomial increases, the convergence goes on faster and faster for all iterations (Picard's, Mann and Ishikawa). 


\section{REFERENCES}

[1] G.V.R Babu and K.N.V.V. Vara Prashad, "Mann iteration converges faster than Ishikawa iteration for the class of Zamfirescu Operators", Fixed Point Theory and Applications, Vol. 2006, Art. ID 49615, 6 pages.

[2] V. Berinde, "Picard's iteration converges faster than Mann iteration for a class of Quasi-Contractive Operators", Fixed Point Theory and Application, Vol. 2004, No. 2. 97-105.

[3] Y. S. Chauhan, R. Rana and A. Negi, "New Julia Sets of Ishikawa Iterates", International Journal of Computer Applications 7(13):34-42, October 2010.

[4] K. Deimling, "Nonlinear Functional Analysis", Springer Verlag, Berlin, 1985.

[5] S. Ishikawa, "Fixed points by a new iteration method", Proc. Amer. Math. Soc.44 (1974), 147150.
[6] W. R. Mann, "Mean value methods in iteration", Proc. Amer. Math. Soc. 4(1953), 506-510.

[7] R. Rana, Y. S. Chauhan and A. Negi, Non Linear dynamics of Ishikawa Iteration, International Journal of Computer Applications 7(13):43-49, October 2010.

[8] B. E. Rhoades and S. M. Soltuz, "The convergence of mean value iteration for a family of maps", I. J. M. M. S., (2005), 3479-3485.

[9] Xue Zhiqun, “ Remarks of equivalence among Picard, Mann and Ishikawa iterations in Normed Spaces", Fixed Point Theory and Applications, Vol. 2007, ArticleId 61434, 5 pages.

[10] Xue Zhiqun, "On the convergence between Mann iteration and Ishikawa iteration for the Generalized

Lipschitizan and $\phi$-strongly pseudocontractive mappings", Bull. Korean Math. Soc. 45 (2008), No. 4, pp- 635-644. 